\title{
Haploinsufficiency of ANKRD11 (16q24.3) Is Not Obligatorily Associated with Cognitive Impairment but Shows a Clinical Overlap with Silver-Russell Syndrome
}

\author{
S. Spengler ${ }^{a} \quad$ B. Oehl-Jaschkowitz ${ }^{b} \quad$ M. Begemann ${ }^{a} \quad$ P. Hennes ${ }^{c} \quad$ K. Zerres ${ }^{a}$ \\ T. Eggermann ${ }^{\text {a }}$ \\ ${ }^{\mathrm{a}}$ Institut für Humangenetik, RWTH Aachen, Aachen, ${ }^{\mathrm{b}}$ Gemeinschaftspraxis für Humangenetik and \\ 'Pädiatrische Endokrinologie, Universitätskinderklinik, Universität des Saarlandes, Homburg, Germany
}

\section{Key Words}

ANKRD11 gene $\cdot$ KBG syndrome · Molecular karyotyping ·

$16 q 24.3$ microdeletion $\cdot$ Silver-Russell syndrome

\begin{abstract}
Microdeletions in $16 q 24.3$ are associated with intellectual disability and a specific phenotype, e.g. short stature and a prominent forehead. The $16 \mathrm{q} 24.3$ microdeletion syndrome shows a broad phenotypic overlap with the KBG syndrome, which is caused by mutations within the ANKRD11 gene. Furthermore, both KBG and the 16q24.3 microdeletion syndromes show clinical findings reminiscent of Silver-Russell syndrome (SRS), an imprinting disorder characterized by severe primordial growth retardation. In a cohort of patients referred as SRS, we previously identified a 16q24.3 deletion, but at that time, only patients with larger imbalances in $16 q 24.3$ and intellectual disability had been published. Considering the recent description of the ANKRD11 gene as the causative factor for the 2 16q24.3-associated disorders, we now classified our patient as a 16q24.3 microdeletion syndrome patient exhibiting some characteristic features but normal intelligence. Our case illustrates the broad clinical spectrum associated with microdeletions, and we confirm that the 16q24.3 microdeletion syndrome is a further microdeletion syndrome with very variable expressivity. Indeed,
\end{abstract}

our case is the first 16q24.3 patient of normal intelligence, but we assume that this variant is present in further mentally healthy probands which have not yet been tested. In conclusion, the detection of the 16q24.3 deletion in a proband of unremarkable intellectual capacities once again illustrates the need to perform molecular karyotyping in dysmorphic patients with normal intelligence.

Copyright $\odot 2013$ S. Karger AG, Basel

Microdeletions in 16q24.3 are by now well known to be associated with intellectual disability and autistic spectrum disorders [for review, see Sacharow et al., 2012]. The deletion carriers show a specific phenotype, e.g. short stature, prominent forehead, arched eyebrows, large ears, and a pointed chin, prompting several groups to suggest a new microdeletion syndrome [Youngs et al., 2011; Isrie et al., 2012; Sacharow et al., 2012] (table 1). In the meantime, heterozygous point mutations within the ANKRD11 gene have been identified in patients with the KBG syndrome (OMIM 148050) [Sirmaci et al., 2011]. The KBG syndrome shows a broad phenotypic overlap with the $16 q 24.3$ microdeletion syndrome, as it is characterized by intellectual disability, short stature, cryptorchidism, clinodactyly $\mathrm{V}$, and distinct craniofacial findings (table 1). Further features include macrodontia of the upper cen-

\section{KARGER}

E-Mail karger@karger.com

www.karger.com/msy
(C) 2013 S. Karger AG, Basel

1661-8769/13/0045-0246\$38.00/0
Thomas Eggermann

Institute of Human Genetics

Pauwelsstr. 30

DE-52074 Aachen (Germany)

E-Mail teggermann@ukaachen.de 
Table 1. Comparison of the clinical features in patients with SRS, KBG syndrome, 16q24.3 microdeletions, and our patient

\begin{tabular}{|c|c|c|c|c|}
\hline Number of patients & 143 & 7 out of 5 families & 9 out of 8 families & \\
\hline \multirow[t]{2}{*}{ Reference } & Wollmann et al., & for review, see & for review, see & \\
\hline & 1995 & Sirmaci et al., 2011 & Sacharow et al., 2012 & \\
\hline IUGR & $94 \%$ & no & no & yes \\
\hline Short stature & $95 \%$ & $6 / 7$ & $8 / 9$ & yes \\
\hline Rel. macrocephaly & $64 \%$ & no & no & yes \\
\hline Micrognathia & $44 \%-73 \% *$ & no & no & no \\
\hline Pointed chin & no & $2 / 7$ & $6 / 9$ & yes \\
\hline Ear anomalies & $53 \%$ & $7 / 7$ & $3 / 9$ & yes \\
\hline Seizures & no & $2 / 7$ & no & no \\
\hline \multicolumn{5}{|l|}{ Other features } \\
\hline Asymmetry & $51 \%$ & no & no & no \\
\hline Clinodactyly V & $68 \%$ & $7 / 7$ & $2 / 9$ & yes \\
\hline Cryptorchidism & no & $6 / 7$ & $2 / 7$ & no \\
\hline Heart defects & no & no & $2 / 8$ & no \\
\hline
\end{tabular}

Clinical signs characterized for SRS and/or KBG syndrome are printed in bold face. IUGR = Intrauterine growth retardation; ID = intellectual disability; ADHD = attention deficit hyperactivity disorder. ${ }^{*}$ Data on the frequency of micrognathia were obtained from Kotzot [2008], who separated them into the molecular subgroups.

tral incisors and skeletal anomalies. Considering the clinical features in both KBG and 16q24.3 microdeletion syndromes, a central role of ANKRD11 in the craniofacial and central nervous system development and function has been postulated [Sirmaci et al., 2011].

Both KBG and the 16q24.3 microdeletion syndromes show clinical findings reminiscent of Silver-Russell syndrome (SRS, OMIM 180860). SRS is a congenital imprinting disorder typically associated with mutations and epimutations in chromosomes 7 or 11p15 [for review, see Abu-Amero et al., 2008] (table 1). However, several key features of SRS are unspecific, e.g. intrauterine growth retardation, short stature and clinodactyly V, thereby making the clinical diagnosis difficult. As a result, there is a significant clinical overlap with other congenital syndromes, and patients with the initial clinical diagnosis of SRS could be identified as carriers of other known microdeletion syndromes, e.g. 1q21, 12q14, $15 \mathrm{q} 26,17 \mathrm{p} 13$, and 22q11 [for review, see Bruce et al., 2010; Spengler et al., 2012].

ANKRD11 Deletion in Silver-Russell Syndrome
In a cohort of patients referred as SRS but without the classical chromosome 7 and 11 p15 alterations, we previously identified a patient carrying a deletion in $16 \mathrm{q} 24.3$ [patient SR596/07 in Spengler et al., 2012], but at that time, only patients with larger overlapping imbalances in 16q24.3 and intellectual disability had been published. Therefore, we classified that patient as carrier of a copy number variation of unknown significance. Considering the recent description of the ANKRD11 gene as the causative factor for both the KBG and the 16q24.3 microdeletion syndrome, we now reevaluated our clinical data on this patient.

\section{Patient}

The patient is the third child of a healthy, consanguineous Turkish couple (father's height: $176 \mathrm{~cm}$, mother's height: $157 \mathrm{~cm}$ ). The pregnancy was uneventful, and the boy was born at term after Caesarean section because of fetal malposition. Birth weight was 2,690 g (SD -1.84), length $49 \mathrm{~cm}(\mathrm{SD}-1.26)$ and head circumfer- 

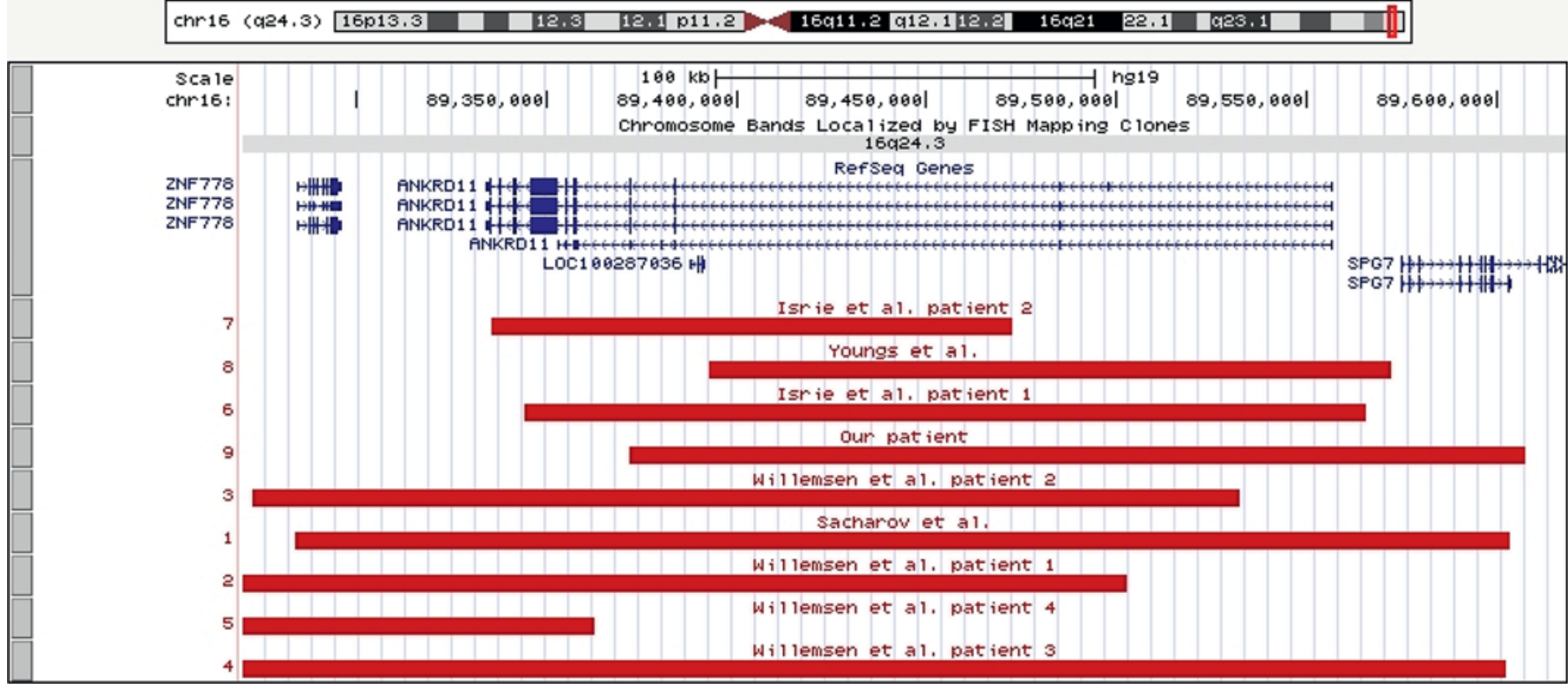

Fig. 1. Detailed view of the UCSC genome browser custom tracks of alterations in patients with 16q24.3 microdeletions, including our patient. Regions affected in the known microdeletion syndromes are shown in dark red. Genes probably associated with the patients' phenotype are highlighted in blue.

ence $35 \mathrm{~cm}(\mathrm{SD}-0.23)$ (table 1$)$. The postnatal period was uneventful. At the age of $910 / 12$ years, he was referred to genetic testing because of short stature [122 cm (SD -2.22), $23.4 \mathrm{~kg}$ (BMI 15.7)]. Growth retardation persisted at the age of 13 years $[142 \mathrm{~cm}$ (SD $-1.73)]$. Head circumference was increased $(53.5 \mathrm{~cm}(\mathrm{SD}+0.29)$ at $910 / 12$ years, $56.5 \mathrm{~cm}(\mathrm{SD}+1.74)$ at 13 years $)$, a finding compatible with relative macrocephaly. In addition to his short stature, the patient showed a prominent forehead, a triangular face with a pointed chin, a prominent nose with a high nasal bridge, large, dysplastic and low-set ears, and enlarged upper incisors. Clinodactyly V, cryptorchidism or congenital heart defects as typical symptoms of KBG/16q24.3 microdeletion syndromes were not observed, although a mild clinodactyly $\mathrm{V}$ was noted in earlier reports. Psychomotoric development was normal. He currently visits a secondary school with moderate marks. Formal intelligence tests were not performed.

As reported recently [Spengler et al., 2012], molecular karyotyping with an Affymetrix GeneChip ${ }^{\circledR}$ Genome-Wide Human SNP 6.0-Array revealed a heterozygous de novo 348.4-kb deletion in 16q24.3 (hg19: chr16:89,371,838-89,607,414) in our patient (fig. 1).

\section{Discussion}

The association of $16 \mathrm{q} 24.3$ microdeletions and $A N$ KRD11 mutations with intellectual disability, postnatal growth retardation and specific craniofacial dysmorphisms has recently been documented by several studies [for review, see Simarci et al., 2011; Sacharow et al., 2012], and the overlapping KBG and 16q24.3 microdeletion syndromes have been delineated. Interestingly, some of these features overlap with SRS, e.g. growth retardation, a triangular face and clinodactyly of the fifth digit. By searching for genomic imbalances in patients referred as SRS, we previously identified a carrier of a heterozygous $348.4-\mathrm{kb}$ deletion in 16q24.3 (including the ANKRD11 gene) [Spengler et al., 2012]. At that time, information on further 16q24.3 carriers was sparse, and we, therefore, could not classify our findings. With the recent identification of ANKRD11 suggested as the disease-causing gene both in 16q24.3 microdeletion and KBG syndromes, we reevaluated our data of the aforementioned patient. On genomic DNA level, the deletion spans $384.4 \mathrm{~kb}$ and includes the ANKRD11 gene itself and a part of SPG7. Similar cases have been reported in the literature exhibiting the 16q24.3 microdeletion phenotype (fig. 1). The initial clinical diagnosis of SRS in our patient was mainly based on the postnatal growth retardation, the relative macrocephaly and the triangular face. Indeed, these clinical findings can also be attributed to the $16 \mathrm{q} 24.3$ microdeletion, whereas the normal intellectual development as well as the macrocephaly do not correspond to the previously reported 16q24.3 microdeletion phenotype [Sacharow et al., 2012]. 
In total, our case impressively illustrates the broad clinical spectrum associated with microdeletions, and we suggest that the 16q24.3 microdeletion syndrome should be classified as a further microdeletion syndrome with variable expressivity. Interestingly, our case is the first 16q24.3 microdeletion carrier with normal intelligence, but we assume that this variant is present in further probands with normal intellect. These carriers currently escape detection, as molecular karyotyping is focused on patients with intellectual disability.

As several groups have shown, the SRS phenotype shows a broad overlap with other congenital syndromes, and - as a result - different microdeletions have been re- ported in SRS patients [for review, see Bruce et al., 2010; Spengler et al., 2012]. In conclusion, the detection of a 16q24.3 deletion in a proband of unremarkable intellectual capacities once again illustrates the need to perform molecular karyotyping in patients with dysmorphisms but without intellectual disability and to critically reassess their clinical data.

\section{Acknowledgement}

We thank the family for participating in this study. The project was supported by the Bundesministerium für Bildung und Forschung (Network 'Imprinting Diseases', 01GM1114C).

\section{References}

Abu-Amero S, Monk D, Frost J, Preece M, Stanier P, Moore GE: The genetic aetiology of SilverRussell syndrome. J Med Genet 45:193-199 (2008).

Bruce S, Hannula-Jouppi K, Puoskari M, Fransson I, Simola KO, et al: Submicroscopic genomic alterations in Silver-Russell syndrome and Silver-Russell-like patients. J Med Genet 47:816-822 (2010).

Isrie M, Hendriks Y, Gielissen N, Sistermans EA, Willemsen $\mathrm{MH}$, et al: Haploinsufficiency of ANKRD11 causes mild cognitive impairment, short stature and minor dysmorphisms. Eur J Hum Genet 20:131-133 (2012).
Kotzot D: Maternal uniparental disomy 7 and Silver-Russell syndrome - clinical update and comparison with other subgroups. Eur J Med Genet 51:444-451 (2008).

- Sacharow S, Li D, Fan YS, Tekin M: Familial 16q24.3 microdeletion involving ANKRD11 causes a KBG-like syndrome. Am J Med Genet 158A:547-552 (2012).

-Sirmaci A, Spiliopoulos M, Brancati F, Powell E, Duman D, et al: Mutations in ANKRD11 cause KBG syndrome, characterized by intellectual disability, skeletal malformations, and macrodontia. Am J Hum Genet 89:289-294 (2011).

- Spengler S, Begemann M, Ortiz Brüchle N, Baudis M, Denecke B, et al: Molecular karyotyping as a relevant diagnostic tool in children with growth retardation with Silver-Russell features. J Pediatr 161:933-942 (2012).
Willemsen MH, Fernandez BA, Bacino CA, Gerkes E, de Brouwer AP, et al: Identification of ANKRD11 and ZNF778 as candidate genes for autism and variable cognitive impairment in the novel 16q24.3 microdeletion syndrome. Eur J Hum Genet 18:429-435 (2010).

Wollmann HA, Kirchner T, Enders H, Preece MA, Ranke MB: Growth and symptoms in Silver-Russell syndrome: review on the basis of 386 patients. Eur J Pediatr 154:958-968 (1995).

Youngs EL, Hellings JA, Butler MG: ANKRD11 gene deletion in a 17-year-old male. Clin Dysmorphol 20:170-171 (2011). 